\title{
Herramientas virtuales de comunicación como experiencia innovativa de aprendizaje en la Tecnicatura en Turismo Rural de la FAUBA
}

\section{Fernández, Sandra a , Deluca, Djasmine ${ }^{b}$}

${ }^{a}$ Facultad de Agronomía, Universidad de Buenos Aires (FAUBA), b aFacultad de Agronomía, Universidad de Buenos Aires (FAUBA).

\begin{abstract}
This work aims to document the experience of technological innovation implemented in the Technique of Rural Tourism in the Faculty of Agronomy of the UBA. The space provided as a formative proposal, through encouraging own productions in social networks, encourages immersive practice in which students propose a product and launch it into the virtual world without intermediation. This practice has been perfected over the years and the results are very encouraging. We present here TWO of the works presented during the year 2018 as representative cases of the course taught in the subject "Taller 1". In addition to the basic training, developed with the guidance of the teacher, students learn to use tools of greater sophistication and complexity that are valued as important skills for their professional development.
\end{abstract}

Keywords: Social networks, University education, Territories, Rural Tourism, Technology education project

\footnotetext{
Resumen

Este trabajo se propone documentar la experiencia de innovación tecnológica implementada en la Tecnicatura de Turismo Rural en la Facultad de Agronomía de la UBA. El espacio suministrado como propuesta formativa, a través de incentivar las producciones propias en redes sociales, fomenta la práctica inmersiva en la que los estudiantes proponen un producto y lo lanzan al mundo virtual sin intermediaciones. Esta práctica se ha perfeccionado a través de los años y los resultados son muy alentadores. Presentamos aquí DOS de los trabajos presentados durante el año 2018 a modo de casos representativos del curso dictado en la asignatura Taller 1. Además del entrenamiento básico, desarrollado con acompañamiento del docente, los estudiantes aprenden a manejar herramientas de mayor sofisticación y complejidad que son valoradas como capacidades importantes para su desarrollo profesional.
}

Palabras clave: Redes sociales, Enseñanza universitaria, Territorios, Turismo Rural, Proyecto de educación con tecnología. 


\section{Introducción}

El presente trabajo se enmarca en la implementación de experiencias innovativas desarrolladas en la asignatura Taller 1 de la Tecnicatura en Turismo Rural de la Facultad de Agronomía de la Universidad de Buenos Aires.

El desarrollo actual del turismo se caracteriza por presentar una dinámica de crecimiento que ha modificado notablemente las tendencias pasadas. La observación de una creciente demanda de propuestas recreativas y de ocio por parte del habitante urbano, que ha motivado una tendencia a viajar a lugares naturales y zonas rurales, ponen en valor potencial las labores desarrolladas en el ámbito natural. A su vez, desde el lado de los productores rurales, cuyas actividades se basan en el sector primario de la economía, se pone de manifiesto la exposición a las fluctuaciones de precios, del mercado, de las condiciones climáticas, de las políticas de estado y otros factores, los que influyen directamente en los resultados. La problemática de este sector es compleja y se caracteriza por una dinámica evolutiva que arroja como resultado una tendencia migratoria hacia los centros urbanos, mayoritariamente de la población joven.

La industria turística en estos últimos años se ha establecido como un fenómeno de extraordinaria relevancia económica, cultural y social, por lo cual se han creado dependencias vinculadas al área con el propósito de posicionar este campo como eje estratégico de expansión del país y de la región.

La evolución de las organizaciones turísticas es cada vez más dinámica y compleja. La globalización, la competitividad, los cambios económicos, tecnológicos, legales, ambientales, de mercado, etc, sumados a su crecimiento e integración hacen que la tarea de administración y gestión de los recursos turísticos requiera de profesionales sólidamente formados para el éxito del sector. Las empresas y emprendimientos turísticos necesitan nutrirse de más y mejores elementos para la toma de decisiones, manteniendo una visión de largo plazo en un ambiente internacional que requiere profesionalización y conocimiento de herramientas de gestión y comunicación para alcanzar sus objetivos con eficiencia. A su vez, el medio rural está inmerso en un fenómeno de multifuncionalidad, en el que los recursos naturales disponibles tienen la potencialidad de organizarse en torno a actividades alternativas a la producción agropecuaria tradicional pero dada la naturaleza eminentemente interactiva de la actividad turística, la gestión de herramientas de comunicación cobra importancia vital.

Tomando cuenta de estos fenómenos, la Universidad de Buenos Aires por intermedio de la Facultad de Agronomía ha lanzado en el año 2008 la Tecnicatura en Turismo Rural (TTR), para poner a disposición del sector un cuerpo de profesionales altamente capacitados para administrar racional y responsablemente los recursos dedicados al turismo que ofrece este medio. Este profesional cumple con la capacidad de planear, organizar, dirigir y controlar las actividades de la gestión empresarial como un proceso administrativo que sustente la toma de decisiones, a través de la definición del problema, de la identificación de alternativas, de la evaluación y de la elección de una de ellas, de la consideración de una decisión y de la capacidad individual de controlar los resultados obtenidos. En términos de Brunner (1988) 
se trata de un enfoque pedagógico donde se ponen en juego categorías de imaginación, innovación, creatividad, al servicio de la resolución de problemas.

El turismo es un hecho social irreversible que genera una serie de interrelaciones e intercambios que tienen consecuencias de diversa índole, tanto para los visitantes como para los oferentes de propuestas de turismo rural. Por otro lado, la influencia de las tecnologías comunicacionales imprimen una dinámica del que el sector no permanece ajeno. Ambos fenómenos determinan nuevas formas de relacionarse entre productores y consumidores, en este caso turistas, dando lugar a que las herramientas de uso cotidiano pasen naturalmente a formar parte de las contrataciones, recomendaciones, muestras, es decir del corazón del negocio turístico. Es imprescindible entonces que los estudiantes de esta temática reciban capacitaciones apropiadas para su mejor inserción en el mundo laboral. En términos de Lion (1988) es necesario adecuarnos a las nuevas formas de relacionamiento y fomentar un aula con "porosidades", capaz de intercambiar e integrar información con el medio a través de las herramientas tecnológicas disponibles. Este concepto a su vez refuerza el de "zona de desarrollo proximal" en el que Vigotzky (1988) enfatiza la potencia que adquiere el aprendizaje cuando está enmarcado en un contexto sociocultural conocido por el aprendiz. Este recorrido es fortalecido a su vez cuando está sostenido por un "andamiaje" adecuado, provisto por los docentes encargados de acompañar y tutorear el proceso.

En este sentido, en el marco particular de la asignatura Taller 1 se implementan diversas técnicas didácticas innovadoras, las que evolucionan en función de la vertiginosa aceleración de las tecnologías. Entre ellas, se focalizan en este trabajo las capacidades desarrolladas en el ámbito de las tecnologías de información y comunicación, focalizadas en la conformación de herramientas de comunicación virtual por parte de los alumnos que cursan esta asignatura. Si se toma como referencia el trabajo de Maggio (2012), se trata de "reinventar" la clase, arriesgar a nuevos modos de búsqueda, de creación, de libertad de acción.

Cabe preguntarnos en perspectiva histórica y sectorial ¿cómo nos encuentra hoy el siglo XXI a los universitarios y a las instituciones de educación superior frente a los nuevos paradigmas de gestión y comunicación? ¿Cómo se conforman y gestionan los nuevos modelos institucionales de la formación y de la investigación inmersos en un mundo hiperconectado? ¿Cómo intervenir responsablemente en la construcción de identidades y grupos académicos comprometidos con la innovación y el sostén de una producción de excelencia y a la vez dar respuesta a las demandas sociales, ambientales, éticas, aún en condiciones críticas? Estas cuestiones nos interpelan directamente. Nos alertan y convocan a realizar un ejercicio de autosocioanálisis reflexivo. Nos incitan a un movimiento pendular entre lo trascendente y lo pragmático. Un ejercicio que se nos impone complejo desde la partida: actuar responsablemente en referencia al modo en que los universitarios nos vemos implicados, al constituir/constituirnos en objeto de estudio a nosotros mismos, nuestra labor académica y las instituciones donde nos desempeñamos. En definitiva, estamos siendo universitarios que investigamos a los universitarios; sus (nuestras) prácticas, trayectorias, representaciones, producciones, espacios de socialización, con el riesgo que ello implica. Dichas marcas cobran visibilidad a través de nuestras acciones, nuestras ideas y nuestras producciones. Emergen y se expresan mediante múltiples manifestaciones: reacciones inmediatas y capacidad de 
organización ante requerimientos inesperados; respuestas individuales o colectivas con un alto grado de resolución; capacidad de adaptación o a la inversa, de resistencia, a cambios estructurales radicales. Sobre estas cuestiones, vinculadas a la incorporación de actividades innovativas en el aula, se propone discutir este trabajo.

\section{Objetivos}

Incorporar herramientas innovativas de comunicación virtual por parte de los alumnos de la asignatuta Taller 1 para la mejora de sus competencias digitales.

Integrar en la currícula nuevas prácticas de aprendizaje con sentido inmersivo y perspectiva tecnológica para la inserción competitiva del futuro profesional.

\section{Desarrollo de la Innovación}

En el contexto presentado precedentemente, la Tecnicatura en Turismo Rural la Facultad de Agronomía de la Universidad de Buenos Aires, se presenta como una carrera de tres años de duración, caracterizada por materias que maryormente adoptan un formato teórico práctico. La materia Taller 1 es una asignatura anual correspondiente al primer año de la carrera que contiene un módulo dedicado a las TIC denominado "Recursos Tecnológicos de Comunicación Virtual”. Dicho módulo se incluye en la asignatura desde el inicio de la carrera $\mathrm{y}$ con las sucesivas cohortes ha sufrido mutaciones en cuanto a sus contenidos, prácticas, y didáctica, en función de las transformaciones que han sugerido los estudiantes y a los ajustes percibidos como necesarios producto de la evolución tecnológica y la formación de los docentes. En este sentido, la tradicional progresión lineal ha sufrido modificaciones y se ha aceptado como válido un esquema proyectual de creación propia e interacción con el medio como alternativa favorable al aprendizaje (Burbules, 2001).

El formato proyectual de la propuesta resultó apropiado para la construcción del conocimiento y la consolidación progresiva del proceso de aprendizaje. A través de ejercicios prácticos distribuidos en los dos cuatrimestres se propiciaron espacios y tiempos suficientes para la participación de los alumnos como sujetos activos en el proceso de aprendizaje.

Es así mismo importante destacar que uno de los rasgos característicos de los matriculados consiste en la heterogeneidad en rango etario, nivel educativo previo, procedencia geográfica e intereses profesionales. Esta diversidad se refleja en las producciones finales del módulo donde se ponen en juego los diferentes lenguajes, elementos simbólicos y grupos de intercambio con la comunidad social.

El dictado del módulo "Recursos Tecnológicos de Comunicación Virtual" durante el 2018 constó de cuatro encuentros presenciales a lo largo del año y de un seguimiento permanente mediante una plataforma digital de aprendizaje. 
Durante el primer encuentro se efectuó una presentación general y puesta en común a partir de un material de contextualización sobre los alcances, implicancias y tipos de recursos tecnológicos de comunicación virtual que aplican actualmente en el ámbito del Turismo Rural. Se presentaron casos reales que utilizan las plataformas Instagram, Facebook, Wordpress, abriendo el debate sobre su diseño y gestión de contenidos como activo estratégico de comunicación.

Durante el segundo encuentro se establecieron las consignas del trabajo práctico. Cada alumno, en forma individual o en pares, escogió uno de los soportes tecnológicos disponibles para diseñar y crear contenidos. La clase se llevó a cabo en aula informática, con ordenadores dispuestos en $\mathrm{U}$, asistidos por el docente en los primeros pasos de cada proyecto. Asímismo se ofrecieron tutoriales y guías de requerimientos mínimos pretendidos de redacción, de nivel estético, de cantidad de publicaciones, así como de plazos y modos de entrega final.

En los siguientes DOS meses cada alumno o pares de alumnos construyó sus contenidos asistidos mediante un seguimiento en forma remota, con la consigna de compartir la progresión de las producciones en un foro de consulta común entre alumnos y docente.

Por último, hacia fines del segundo cuatrimestre, todos los proyectos fueron exhibidos en una exposición oral y visual. Se mostraron capturas de pantalla, interacciones reales, recursos creativos y novedosos, destacando especialmente los detalles del proceso para compartir la experiencia.

Al concluir la clase se ofreció una breve encuesta anónima que permitió la retroalimentación con la voz de los alumnos en cuanto a nivel de motivación, valoración de las herramientas aprendidas, nivel de dificultad que presentó el proyecto, apreciación de los conocimientos y rol del docente a cargo.

Se realizaron CATORCE producciones. OCHO de ellas elaboradas en la plataforma global del gestor de contenidos WordPress; CINCO en la red social Instagram y UNA en la red social Facebook. Los trabajos logrados, el proceso de producción y toma de decisiones, y las experiencias compartidas por los alumnos en el cierre del proyecto ejemplifican la contundencia positiva del modelo de introducción de estas prácticas tecnológicas en el ámbito académico.

A modo ilustrativo se exponen DOS de las producciones resultantes del grupo que cursó la asignatura durante el año 2018, seleccionadas por destacarse en cuanto a nivel estético, uso apropiado de herramientas del soporte, creatividad y relevancia de la temática elegida, como por el adecuado recorrido y evolución del proyecto.Así mismo, la elección obedece a que durante el mencionado período lectivo la práctica pedagógica ha madurado con respecto a los ciclos anteriores y se ha plasmado en trabajos que expresan significativamente lo que se quiere mostrar en este artículo.

\subsection{Aviturismo Argentina}

Soporte: WordPress

Alumnos: Barceló, Mariana; Hermosilla Esquivel, Owen. 
Herramientas virtuales de comunicación como experiencia innovativa de aprendizaje en la Tecnicatura en Turismo Rural de la FAUBA

Descripción: "Brindamos información sobre las especies de aves existentes en la Argentina y lugares de avistaje en su territorio."

Enlace: www.aviturismoargentina.wordpress.com

Principales Etiquetas: Avistaje, Aves, Argentina, Turismo, Naturaleza.

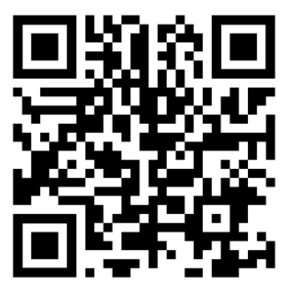

Fig. 1: Código QR de acceso a Aviturismo Argentina.

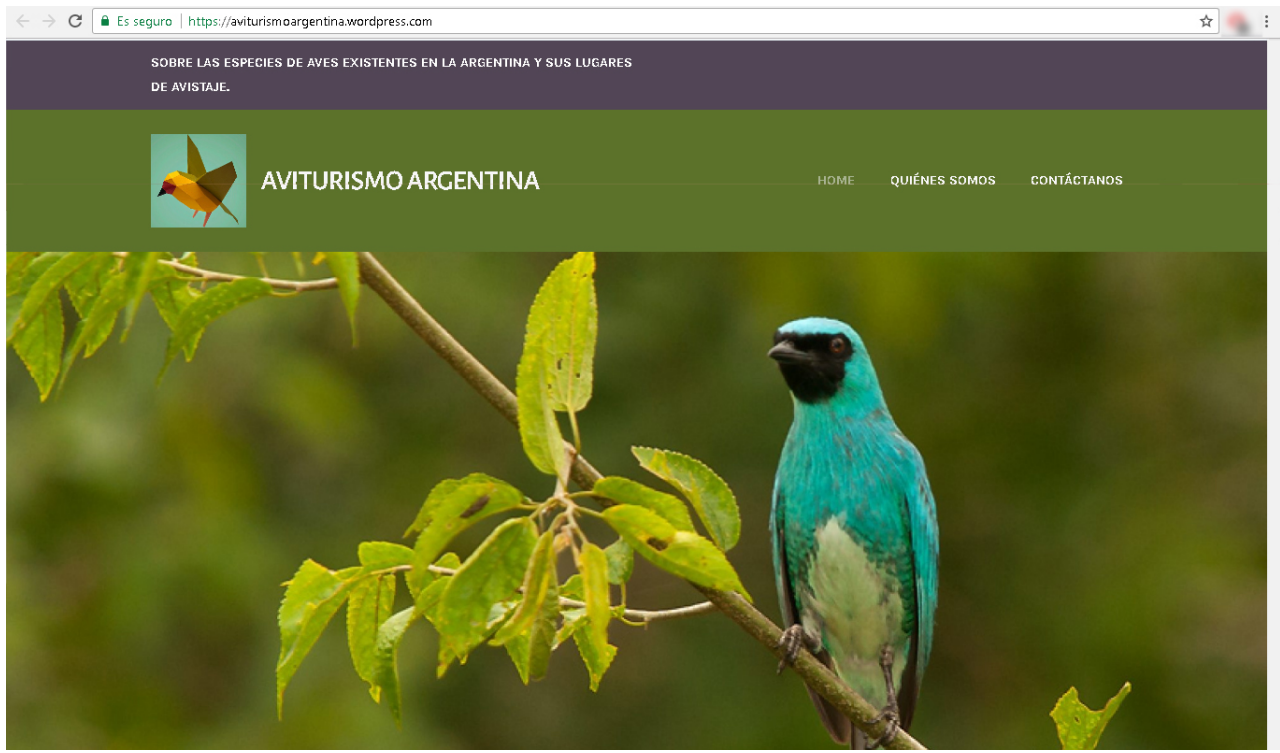

Fig. 2: Captura de pantalla del encabezado del sitio.

Los alumnos eligieron trabajar en la plataforma global del gestor de contenidos WordPress, desarrollando publicaciones sobre el avistaje de aves en Argentina, en su vinculación con las potencialidades que trae esta práctica entre las actividades de Ecoturismo. 


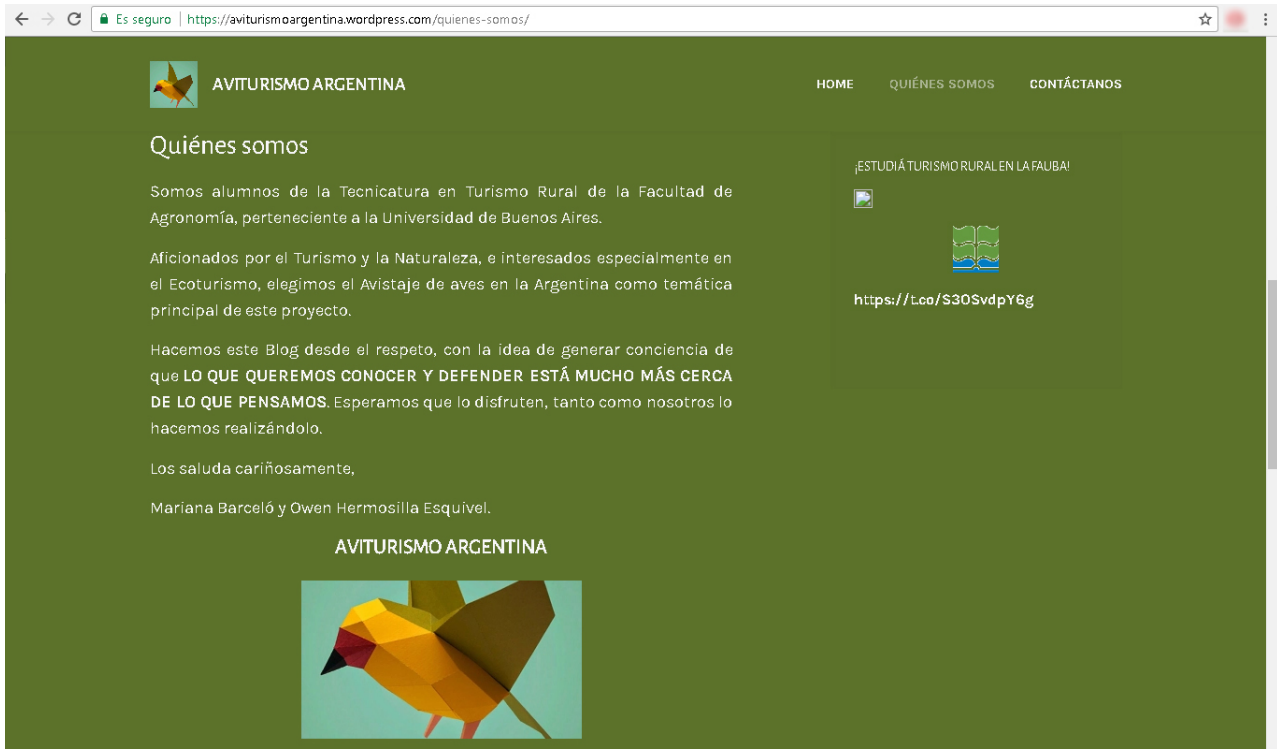

Fig. 3: Captura de pantalla de la sección "Quienes somos".

Entre las herramientas del soporte emplearon las secciones "Quienes Somos" y "Contacto", introduciendo información sobre los autores y el vínculo con la página oficial de la Tecnicatura en Turismo Rural.

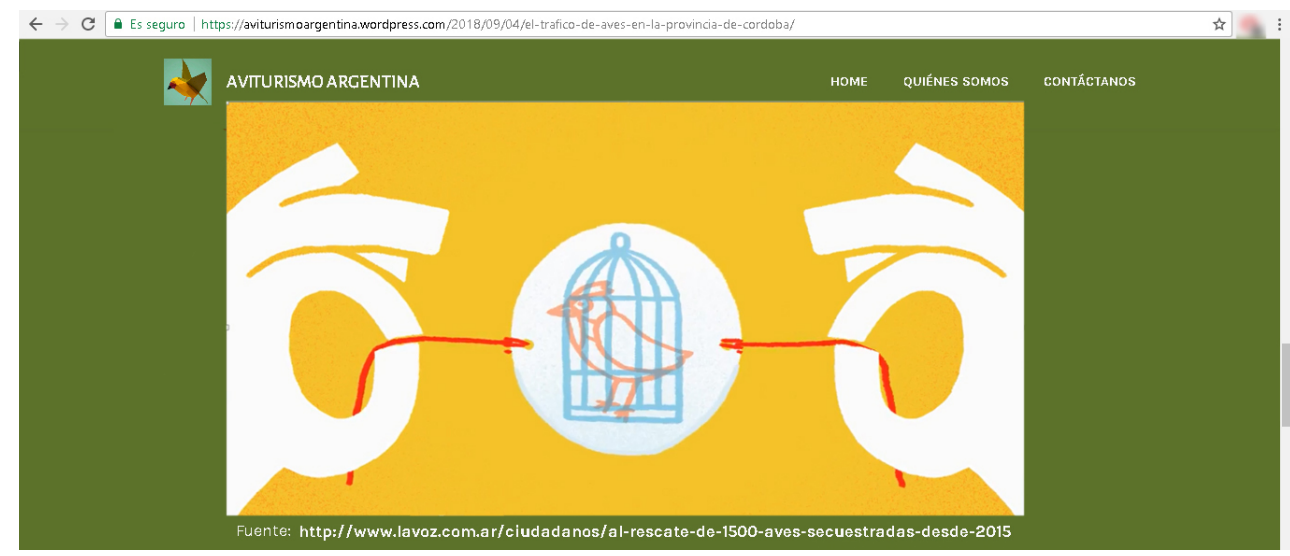

Fig. 4: Captura de pantalla de contenidos.

Se destaca la aplicación de atributos visuales de alto nivel estético, fotos, videos y el empleo de información de excelente redacción. Logrando captar lectores activos dedicados a la observación y protección de aves, con vistas de 8 países diferentes. Son, en este sentido, muy relevantes los comentarios recibidos de especialistas, los cuales dan cuenta de la fiabilidad y alto valor de la información publicada. 


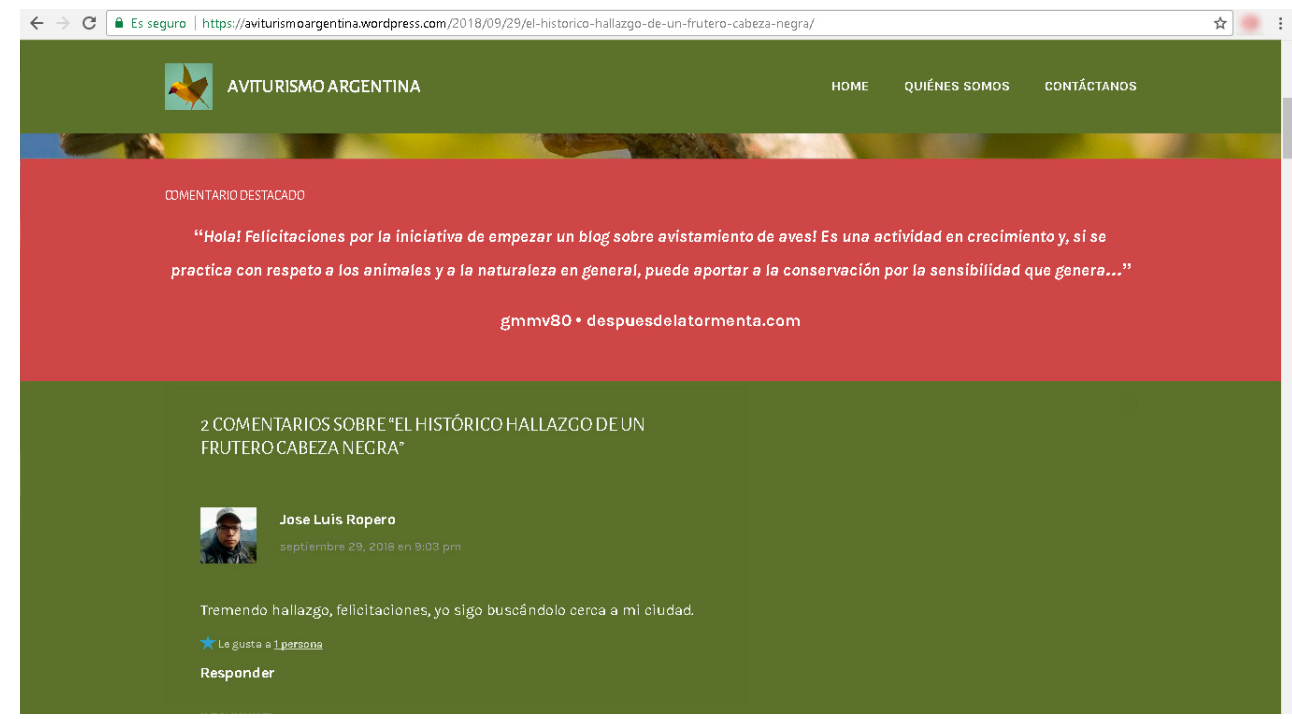

Fig. 5: Captura de pantalla de comentarios de visitantes.

Entre los comentarios finales expuestos por los alumnos mencionan el aprendizaje adquirido al utilizar una herramienta que desconocían hasta el momento del proyecto y la gran motivación que implicó abordar una temática de interés personal.

\subsection{MochilArgentina}

Soporte: Instagram

Alumnos: Huenchuman, Iván.

Descripción: "Te dejamos los mejores lugares de Argentina-Chile con información para guiarte. Animate y vivi otras experiencias, con quien quieras.."

Enlace: www.instagram.com/mochilargentina

Principales hashtags: \#mochilerosarg, \#mochileroschile, \#destinomochilero, \#adnmochilero, \#camping, \#turismorural, \#Argentina, \#Mapuche.

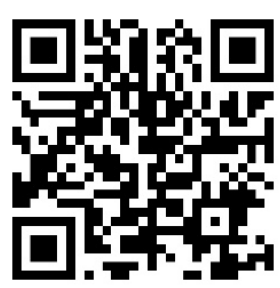

Fig. 1: Código QR de acceso a Mochilargentina. 


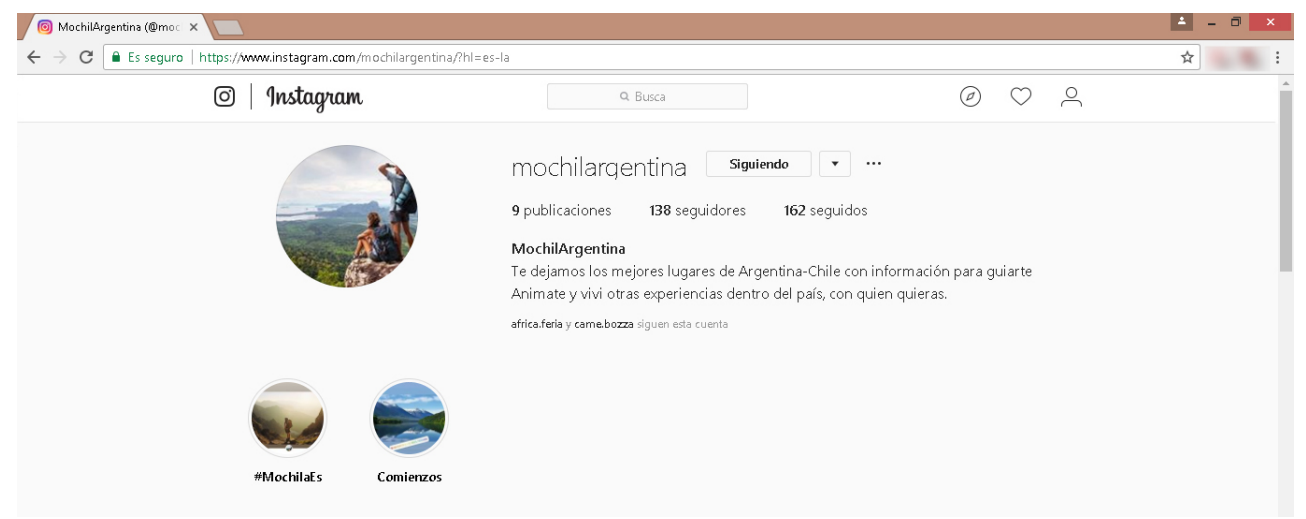

Fig. 5: Captura de pantalla de la información del perfil.

El alumno desarrolló su proyecto empleando la red social Instagram, bajo el usuario @-mochilargentina. En su temática abordadó destinos de Argentina y Chile para "mochileros". Su objetivo principal fue ofrecer información para personas que realizan viajes con su mochila a cuestas y de forma independiente, habitualmente asociadas a practicas de senderismo y campamento.

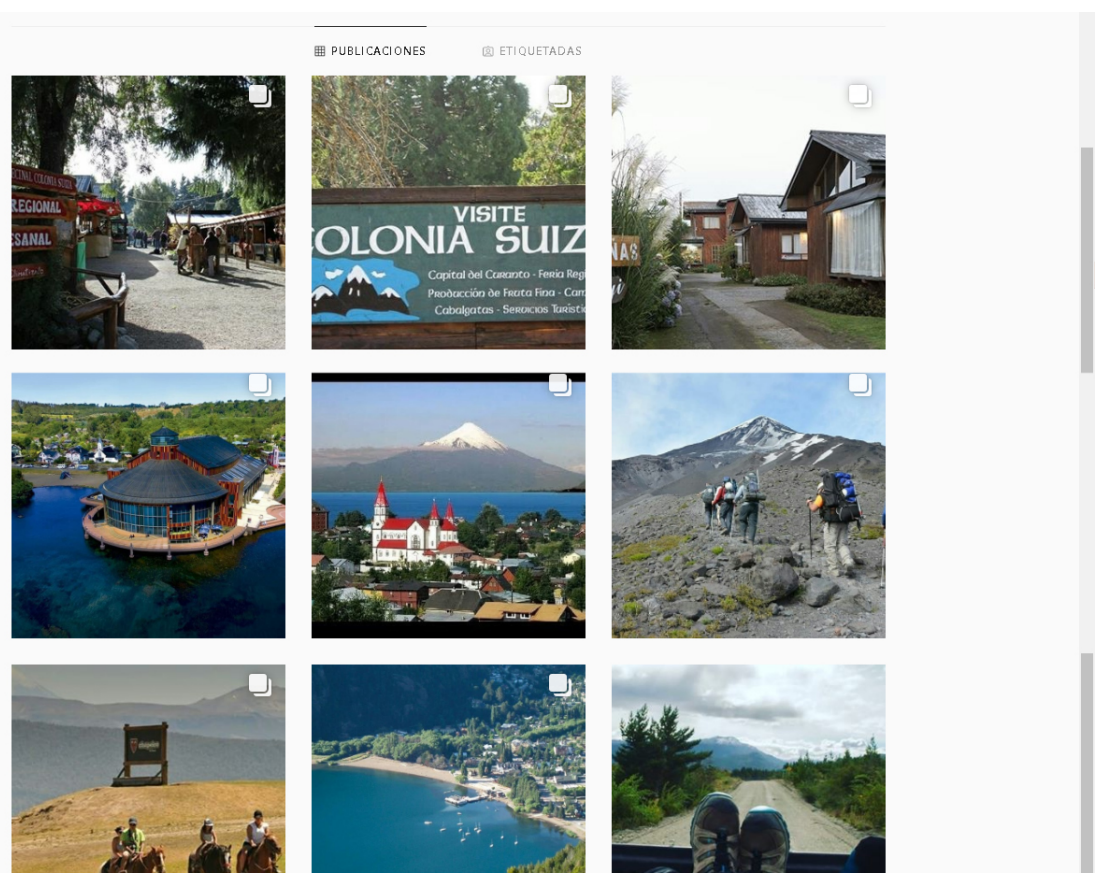

Fig.6: Captura de pantalla de la vista de publicaciones.

Sus publicaciones ofrecen infomación recabada de su propia experiencia personal en la zona patagónica de ambos países. Hecho que no es casual, debido a que se trata de un alumno cuya procedencia geográfica es la Ciudad de Bariloche. Cabe destacar que todos los proyectos se anclaron a territorios concordantes con el lugar de origen de sus autores. 


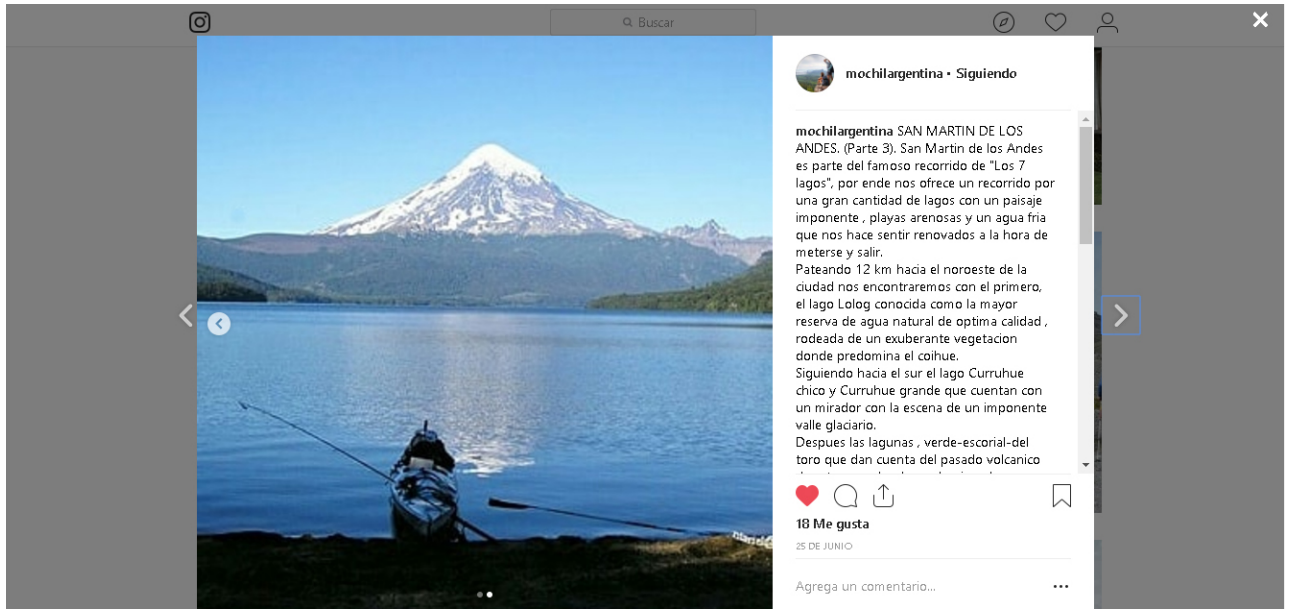

Fig. 7: Captura de pantalla detalle de una publicación.

Aplicó el uso de historias, planteando en ellas situaciones coloquiales y de intrecacción con sus visitantes mediante encuestas, hashtags y consejos para viajeros. Entre los datos mas relevantes comentados se destacan la cantidad de visitantes procedentes de diversos países de habla hispana, así como el amplio espectro etario que comprende desde los 18 años hasta los 60 años. Los datos obtenidos en tiempo real por medio de las estadísticas del soporte superaron las expectativas de interacción del alumno, quien da cuenta del valor que constituyen para estudiar la demanda y gestionar con eficacia las publicaciones

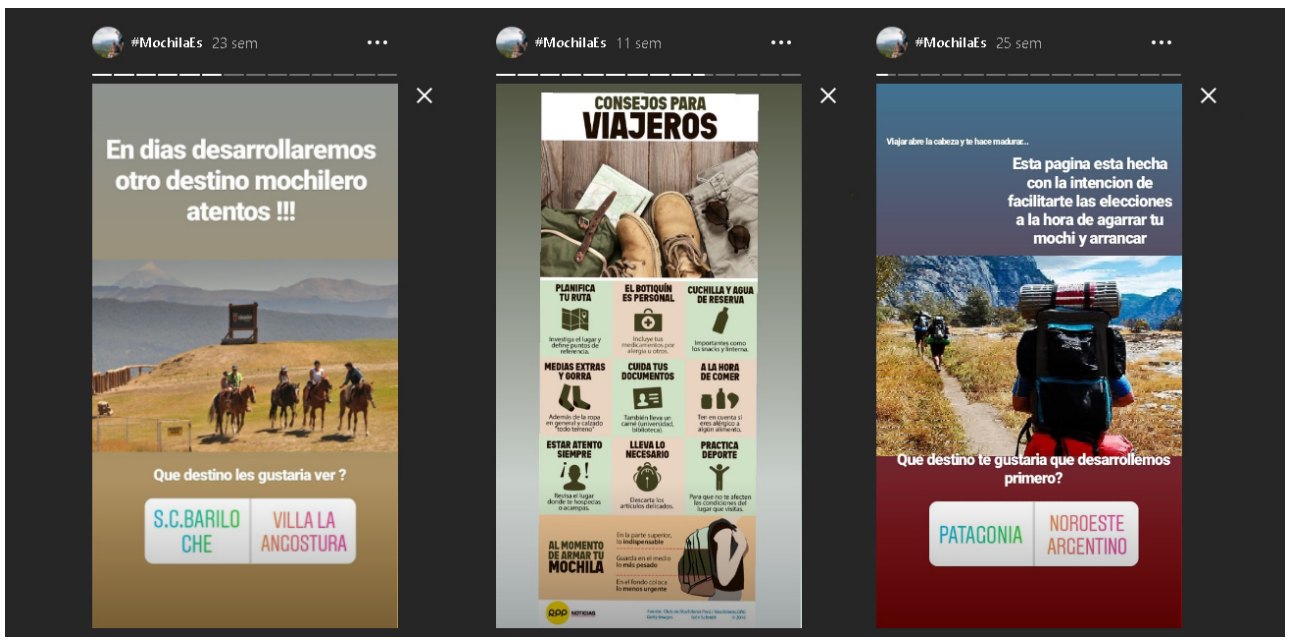

Fig.8: Captura de pantalla de las historias.

.Si bien se toman estos dos ejemplos en mayor profundidad, todos los trabajos producidos abordaron temáticas de anclaje territorial, apropiándose de los soportes elegidos de modos muy diversos. Entre los temas encontramos: Proyectos que desarrollan turismo comunitario, que difunden las áreas protegidas de cada provincia; que brindan información de destinos poco conocidos desde puntos más consolidados en la oferta turística, otros que se basaron en actividades de ecoturismo; que relacionan la vida de personajes y pobladores con lugares de 
interés a partir de entrevistas o historia narradas; así como otros que comparten vivencias personales en diferentes destinos a partir de fotografías, recomendaciones y anécdotas.

\section{Resultados}

A través de la implementación de esta propuesta en Taller 1 se constata que la incorporación de nuevas modalidades de trabajo incentiva a los alumnos a conectarse de modo diferente con su formación y trayectoria de aprendizaje. Se compromete un vínculo con la realidad circundante que emerge en la práctica como facilitador del aprendizaje entendido como construcción.

La interacción con realidades que despiertan interés genuino, como es el caso de la elección del tema para construir la propuesta tecnológica, abre nuevas ramificaciones en el sentido rizomático de Deleuze (2004). Esta categorización implica la generación de un sistema de aprendizaje ramificado, acéntrico, conectado y en el que la estructura se comprende desde la horizontalidad ramificada más que desde la verticalidad.

El rescate de la experiencia por parte de los alumnos recupera el sentido de la apuesta a esta novedosa forma de encarar el aprendizaje universitario. En tal sentido, ambos casos expuestos precedentemente y los restantes DIECISEIS proyectos constituyeron una propuesta de valor a través de la serie de decisiones acertadas que fueron tomando en su proceso de diseño y gestión. Aspectos que se evidencian al rescatar la voz de los alumnos en la encuesta anónima realizada al cierre del proyecto, en la cual el 100\% manifestó que la propuesta les servirá para su desempeño profesional, el 92\% respondió que le interesó y aprendió conceptos, criterios y herramientas nuevas, y el 92\% considera posible continuar aportando material al recurso creado más allá de haber terminado el trabajo práctico.

\section{Conclusiones}

Dentro de esta apuesta del aprendizaje con sentido y perspectiva inmersiva, en términos de la utilización de herramientas tecnológicas con las cuales los estudiantes están familiarizados, se rescatan varios hallazgos para comentar a modo conclusivo. En primer lugar, la valoración altamente positiva de la experiencia como parte de un módulo programático que ubica al estudiante de cara a la sociedad. En este sentido, la práctica propone un rol nuevo: el de la presentación de un producto lanzado al medio social con repercusiones sin intermediaciones, es decir, desacoplado de la experiencia educativa tradicional. Un segundo punto a destacar es que hay una percepción equivocada sobre el conocimiento de las herramientas tecnológicas usadas corrientemente. Los estudiantes conocen lo básico de las herramientas de comunicación virtual (a veces ni siquiera las conocen) y descubren durante el desarrollo del módulo, las potencialidades analíticas, de alcance, de recursos creativos, lo que enriquece sus capacidades de desarrollo profesional. Por último, las producciones presentadas en este trabajo solamente representan una muestra pequeña de las producciones obtenidas, las que cada año se ven enriquecidas por contenidos de mayor creatividad, precisión y repercusión social. 


\section{Referencias}

BRUNNER, J. (1988). Realidad mental y mundos posibles. Barcelona: Gedisa.

BURBULES, A. \&. (2001). Educación: riesgos y promesas de las nuevas tecnologías de la información. Barcelona: Granica.

DELEUZE, G. G. (2004). Mil mesetas. Capitalismo y Esquizofrenia. Valencia: Pre-textos.

LION, C. (2012). Pensar en red: metáforas y escenarios. En M. N. Scialabba, ¿Cómo serán? El futuro de la escuela y las nuevas tecnologías. Buenos Aires: Prometeo.

MAGGIO, M. (5 al 9 de Noviembre de 2012). La clase universitaria re concebida: la creación potenciada por las tecnologías. Webinario UBATIC. I Encuentro virtual sobre TIC y enseñanza en el nivel superior. Buenos Aires, Argentina: CITEP-UBA.

VYGOTSKY, L. (1988). Interacción entre Aprendizaje y Desarrollo. México, México: Grijalbo. 